\title{
APPROXIMATE FUNCTION EXPANSION INTO A CHEBYSHEV SERIES
}

\section{Procedure declaration.}

procedure $T C h e x(p, q, x, f, n, a, e x)$;

value $p, q$;

integer $n$;

real $p, q, x, f$;

Boolean ex;

array $a$;

comment For a given natural number $N \geqslant 2$ the procedure TChex calculates the approximate values

$$
a_{0}^{(n)}, a_{1}^{(n)}, \ldots, a_{n}^{(n)} \quad(n=2,4,8, \ldots, n \leqslant N)
$$

of the coefficients of the expansion of $f(x)$ in the interval $\langle p, q\rangle$ into a series of Chebysher. polynomials of first kind, i.e. it calculates numbers (1) such that for $x \epsilon\langle p, q\rangle$ holds

(2) $f(x) \approx \frac{1}{2} a_{0}^{(n)}+\sum_{k=1}^{n} a_{k}^{(n)} T_{k}\left(\frac{2 x-p-q}{q-p}\right) \quad\left(T_{k}(t)=\cos (k \arccos t)\right)$.

This equality is accurate if $f(x)$ is a polynomial of degree at most $n$. Data:

$p, q$ - interval limits $\langle p, q\rangle$,

$f$ - arithmetical expression with value $f(x)$ depending upon $x$, $n$ - natural number $N$.

Results:

$n$ - upper summation limit in (2), a number of the form $2^{l}$ (l-natural),

$a[0: n]$ - array of approximate values of (1) $\left(a[k]=a_{k}^{(n)}\right.$ for $k=0,1, \ldots, n)$.

Remark: During calculations the variable $n$ assumes successively the values $2,4,8, \ldots$ and for each of them the coefficients of (1) 
are found. The final value of $n$ is always not greater than $N$ but it may depend upon the value of $e x$.

Other parameters:

$x$ - the variable occurring in the 4-th actual parameter,

$e x$ - Boolean expression which may depend upon the actual values of $n, a[0: n]$. If $e x=$ true while doubling the value of $n$ then the procedure calculates the coefficients (1) for the new $n$, if $e x=$ false the calculations are finished.

Remarks:

$1^{\text {o }}$ The coefficients $a_{k}^{(n / 2)}\left(k=0,1, \ldots, \frac{1}{2} n\right)$ may be calculated from

$$
a_{k}^{(n / 2)}= \begin{cases}a_{0}^{(n)}+2 a_{n}^{(n)} & (k=0), \\ a_{k}^{(n)}+a_{n-k}^{(n)} & \left(k=1,2, \ldots, \frac{1}{2} n-1\right), \\ a_{n / 2}^{(n)} & \left(k=\frac{1}{2} n\right) .\end{cases}
$$

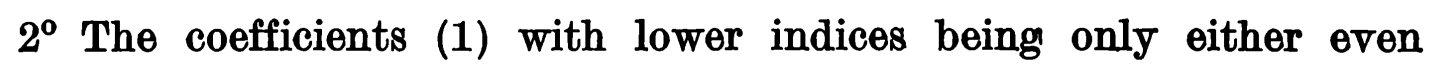
or odd may be obtained from the fact that if we have in the interval $\langle-1,1\rangle$ the formula

$$
g(x) \overline{\overline{d f}} f\left(\frac{1}{2}(p+q)+\frac{1}{2}(q-p) x\right)=\frac{1}{2} a_{0}+\sum_{k=1}^{n} a_{k} T_{k}(x),
$$

then in the interval $\langle 0,1\rangle$ holds

$$
g(\sqrt{x})+g(-\sqrt{x})=a_{0}+2 \sum_{k=1}^{n / 2} a_{2 k} T_{k}(2 x-1)
$$

$$
\sqrt{x}(g(\sqrt{x})-g(-\sqrt{x}))=a_{1}+\sum_{k=1}^{n / 2}\left(a_{2 k-1}+a_{2 k+1}\right) T_{k}(2 x-1) \quad\left(a_{n+1}=0\right)
$$

\section{begin}

integer $h, h 2, i 0, i 1, j, k, m, n 1, n 2$;

real $a 0, a 1 ; a 2, c, c 2$;

$$
\begin{aligned}
& m:=n ; \\
& n:=n 2:=h 2:=2 ; \\
& x:=p ; \\
& a 2:=f ; \\
& x:=p:=.5 \times(p+q) ; \\
& a 1:=f ; \\
& x:=q ; \\
& a 0:=f ;
\end{aligned}
$$




$$
\begin{aligned}
& q:=q-p ; \\
& c:=.5 \times(a 0+a 2) ; \\
& a[0]:=c+a 1 ; \\
& a[1]:=.5 \times(a 0-a 2) ; \\
& a[2]:=.5 \times(c-a 1) ;
\end{aligned}
$$

for $n 2:=n 2+n 2$ while $e x \wedge n 2 \leqslant m$ do

\section{begin}

$$
\begin{aligned}
& n 1:=h 2+1 ; \\
& a 0:=3.14159265359 / h 2 ; \\
& \text { for } j:=h 2 \text { step }-1 \text { until } 1 \text { do } \\
& \text { begin } \\
& \quad c 2:=\cos (a 0 \times(j-.5)) ; \\
& \quad x:=p+q \times c 2 ; \\
& \quad a[h 2+j]:=f
\end{aligned}
$$

end $j$;

$c 2:=c 2+c 2$;

for $h:=h 2$ while $h>1$ do

\section{begin}

$$
\begin{aligned}
& h 2:=.5 \times h ; \\
& a 2:=c:=c 2 ; \\
& c 2:=c 2 \times c 2-2 ;
\end{aligned}
$$

for $j:=0, k$ while $j<h 2$ do

begin

$$
\begin{aligned}
& k:=j+1 \\
& i 1:=n 1-k ; \\
& \text { for } i 0:=n 1+j \text { step } h \text { until } n 2 \text { do } \\
& \quad \text { begin } \\
& \quad i 1:=i 1+h ; \\
& \quad a 0:=a[i 0] \\
& \quad a 1:=a[i 1] ; \\
& \quad a[i 0]:=c \times(a 0-a 1) \\
& \quad a[i 1]:=a 0+a 1 ; \\
& \\
& \quad c:=-c
\end{aligned}
$$


end $i 0$;

$$
\begin{aligned}
& a 0:=a 2 ; \\
& a 2:=a b s(c) ; \\
& c:=c 2 \times a 2-a 0
\end{aligned}
$$

end $j$

end $h$;

$$
\begin{aligned}
& k:=h:=.25 \times n 2 ; \\
& h 2:=n 1-3 ; \\
& \text { for } j:=1 \text { step } 1 \text { until } h 2 \text { do } \\
& \quad \text { begin }
\end{aligned}
$$

if $j<k$

\section{then begin}

$$
\begin{aligned}
& i 0:=n 2-j ; \\
& i 1:=n 2-k ; \\
& a 0:=a[i 0] ; \\
& a[i 0]:=a[i 1] ; \\
& a[i 1]:=a 0 \\
& \text { end } j<k ;
\end{aligned}
$$

$$
i 0:=h \text {; }
$$

$a a:$ if $i 0 \leqslant k$

\section{then begin}

$$
\begin{aligned}
& \quad k:=k-i 0 ; \\
& i 0:=.5 \times i 0 ; \\
& \text { go to } a a \\
& \text { end } i 0 \leqslant k
\end{aligned}
$$

else $k:=k+i 0$

end $j$;

for $h 2:=h$ while $h>1$ do

\section{begin}

$$
\begin{aligned}
& h:=.5 \times h 2 ; \\
& i 0:=n 2-h ;
\end{aligned}
$$

for $k:=i 0-h+1$ step 1 until $i 0$ do 


\section{begin}

$$
\begin{aligned}
& a 0:=a[k] \\
& \text { for } j:=k-h 2 \text { step }-h 2 \text { until } n 1 \text { do } \\
& a 0:=a[j]:=a[j]-a 0
\end{aligned}
$$

end $k$

end $h 2$;

$$
\begin{aligned}
& a 2:=1 / n 2 ; \\
& a[0]:=.5 \times a[0] ; \\
& \text { for } j:=n 1 \text { step } 1 \text { until } n 2 \text { do } \\
& \quad \text { begin }
\end{aligned}
$$

$$
\begin{aligned}
& k:=n 2-j ; \\
& a 0:=.5 \times a[k] ; \\
& a 1:=a 2 \times a[j] ; \\
& a[k]:=a 0+a 1 ; \\
& a[j]:=a 0-a 1
\end{aligned}
$$

end $j$;

$$
\begin{aligned}
& a[0]:=2 \times a[0] ; \\
& n:=h 2:=n 2
\end{aligned}
$$

end $n 2$

end TChex

2. Method used. Let

$$
\begin{aligned}
g(x) & =f\left(\frac{1}{2}(p+q)+\frac{1}{2}(q-p) x\right) \quad(x \in\langle-1,1\rangle), \\
x_{j}^{(n)} & =\cos \frac{j \pi}{n} \quad(n=2,4,8, \ldots ; j=0,1, \ldots, n), \\
\delta_{k n} & = \begin{cases}0 & (k \neq n), \\
1 & (k=n) .\end{cases}
\end{aligned}
$$

Procedure TChex uses the formulae given by Clenshaw [1]

(6)

$$
a_{k}^{(n)}=\frac{2-\delta_{k n}}{n} \sum_{j=0}^{n} g\left(x_{j}^{(n)}\right) T_{k}\left(x_{j}^{(n)}\right) \quad(k=0,1, \ldots, n)
$$

( $\Sigma^{\prime \prime}$ denotes that the first and last summands should be halved) 
to calculate $a_{0}^{(2)}, a_{1}^{(2)}, a_{2}^{(2)}$. All other $a_{k}^{(2 n)}$ are calculated after formulae which follow from (6):

$$
\begin{gathered}
a_{k}^{(2 n)}=\frac{1}{2}\left(a_{k}^{(n)}+b_{k}^{(n)}\right) \quad(k=0,1, \ldots, n-1), \quad a_{n}^{(2 n)}=a_{n}^{(n)}, \\
a_{2 n-k}^{(2 n)}=\frac{1}{2}\left(a_{k}^{(n)}-b_{k}^{(n)}\right) \quad(k=1 ; 2, \ldots, n-1), \quad a_{2 n}^{(2 n)}=\frac{1}{4}\left(a_{0}^{(n)}-b_{0}^{(n)}\right),
\end{gathered}
$$

where $b_{k}^{(n)}$ are expressed by formulae given by Lanczos [3] (p. XVII):

$$
b_{k}^{(n)}=\frac{2}{n} \sum_{j=1}^{n} g\left(x_{2 j-1}^{(2 n)}\right) T_{k}\left(x_{2 j-1}^{(2 n)}\right) \quad(k=0,1, \ldots, n-1) .
$$

Since the numbers given by (7) are Chebyshev coefficients of the polynomial $\boldsymbol{P}$ satisfying

$$
P\left(x_{2 j-1}^{(2 n)}\right)=g\left(x_{2 j-1}^{(2 n)}\right) \quad(j=1,2, \ldots, n),
$$

degree of $P \leqslant n$, therefore, from (5), they are caloulated from the coefficients of the polynomial

and

$$
P_{0}(x)=P\left(\sqrt{\frac{1}{2} x+\frac{1}{2}}\right)+P\left(-\sqrt{\frac{1}{2} x+\frac{1}{2}}\right)
$$

$$
P_{1}(x)=\sqrt{\frac{1}{2} x+\frac{1}{2}}\left(P\left(\sqrt{\frac{1}{2} x+\frac{1}{2}}\right)-P\left(-\sqrt{\frac{1}{2} x+\frac{1}{2}}\right)\right) .
$$

The polynomials $P_{0}$ and $P_{1}$ are of degree at most $\frac{1}{2} n$ and satisfy the following interpolation conditions:

$$
\begin{aligned}
& P_{0}\left(x_{2 j-1}^{(n)}\right)=P\left(x_{2 j-1}^{(2 n)}\right)+P\left(x_{2(n-j)+1}^{(2 n)}\right), \\
& P_{1}\left(x_{2 j-1}^{(n)}\right)=x_{2 j-1}^{(2 n)}\left(P\left(x_{2 j-1}^{(2 n)}\right)-P\left(x_{2(n-j)+1}^{(2 n)}\right),\right.
\end{aligned}
$$

where $j=1,2, \ldots, \frac{1}{2} n$.

If $n=2$, the coefficients of the polynomials $P_{0}$ and $P_{1}$ are already obtained (the coefficient of $T_{n}(x)$ is not needed); otherwise formulae (5) are used repeatedly which leads to $n$ polynomials of degree at most one which satisfy one interpolation condition each.

3. Coefficients of the expansion into a series of Chebyshev polynomials of second kind. If, as before,

$$
g(x)=\frac{1}{2} a_{0}+\sum_{k=1}^{\infty} a_{k} T_{k}(x) . \quad(x \in\langle-1,1\rangle)
$$

and, in addition,

$$
g(x)=\sum_{:=0}^{\infty} \alpha_{k} J_{k}(x) \quad\left(U_{k}(x)=\frac{\sin ((k+1) \arccos x)}{\sqrt{1-x^{2}}}\right),
$$


then it follows from the known relätion $T_{-k}^{\prime}(x)=\frac{1}{2}\left(U_{k}(x)-U_{k-2}(x)\right)$ that

$$
a_{k}=\frac{1}{2}\left(a_{k}-a_{k+2}\right) \quad(k=0,1, \ldots),
$$

and Chebysher coefficients rèlevant to Chebyshev polynomials of 2-nd kind may easily be obtained by procedure TChex.

In addition, the expansion of function $g$.into a series of polynomials $U_{k}(x)$ may be obtained directly using a method similar to that described in Section 2. Thus, for $x \in\langle 0, \overrightarrow{1}\rangle$ the following formulae hold:

$$
g(\sqrt{x})+g(-\sqrt{x})=2 \sum_{k=0}^{\infty}\left(a_{2 k}+a_{2 k+2}\right) U_{k}(2 x-1)
$$

$$
\frac{g(\sqrt{x})-g(-\sqrt{x})}{\sqrt{x}} 4 \sum_{k=0}^{\infty} a_{2 k+1} J_{k}(2 x-1) ;
$$

they are used for calculating $\beta_{k}^{(n)}$, given b.y... (10).

The approximate values

$$
a_{0}^{(n)}, a_{1}^{(n)}, \ldots, \dot{a}_{n}^{(n)} \quad\left(n=2^{l}-2 ; l=1,2, \ldots\right)
$$

of the Chebyshev coefficients are calculated from the recurrent formulae

where

$$
\begin{aligned}
\alpha_{0}^{(0)} & =g(0), \\
\alpha_{k}^{(2 n+2)} & =\frac{1}{2}\left(\beta_{k}^{(\dot{n})}+\alpha_{k}^{(n)}\right) \\
a_{2 n+2-k}^{(2 n+2)} & =\frac{1}{2}\left(\beta_{k}^{(n)}-\alpha_{k}^{(n)}\right) \\
\alpha_{n+1}^{(2 n+2)} & =\frac{1}{2} \beta_{n+1}^{(n)}
\end{aligned}
$$

$$
\begin{array}{r}
\beta_{k}^{(n)}=\frac{2}{n+2} \sum_{j=1}^{n+2}\left(1-\left(x_{2 j-1}^{(2 n+4)}\right)^{2}\right) g\left(x_{2 j-1}^{(2 n+4)}\right) U_{k}\left(x_{2 j-1}^{(2 n+4)}\right) \\
(k=0,1, \ldots, n+1) .
\end{array}
$$

These formulae follow from an application of a known quadrature (see e.g. [2], p. 111, formula (7.3.7)):

$$
\begin{aligned}
a_{k} & =\frac{2}{\pi} \int_{-1}^{1} \sqrt{1-x^{2}} g(x) U_{k}(x) d x \\
\because & \frac{2}{n+2} \sum_{j=1}^{n+1}\left(1-\left(x_{j}^{(n+2)}\right)^{2}\right) g\left(x_{j}^{(n+2)}\right) U_{\vec{k}}^{\prime}\left(x_{j}^{(n+2)}\right)=\alpha_{k}^{(n)} .
\end{aligned}
$$


The numbers $\beta_{k}^{(n)}$, similarly as $b_{k}^{(n)}$ before, are obtained by applying (8) to the polynomial

$$
Q(x)=\sum_{k=0}^{n} \beta_{k}^{(n)} U_{k}(x)+\frac{1}{2} \beta_{n+1}^{(n)} U_{n+1}(x)
$$

and to the polynomials which arise from $Q$ in such a way.

The definition of $Q$ and [2] (p. 111, formula (7.3.6)) yield

$$
\begin{aligned}
\beta_{k}^{(n)} & =\frac{2+2 \delta_{k, n+1}}{\pi} \int_{-1}^{1} \frac{\left(1-x^{2}\right) Q(x) U_{k}(x)}{\sqrt{1-x^{2}}} d x \\
& =\frac{2}{n+2} \sum_{j=1}^{n+2}\left(1-\left(x_{2 j-1}^{(2 n+4)}\right)^{2}\right) Q\left(x_{2 j-1}^{(2 n+4)}\right) U_{k}\left(x_{2 j-1}^{(2 n+4)}\right) \quad(k=0,1, \ldots, n+1),
\end{aligned}
$$

and, because the interpolation polynomial is uniquely determined, there is

$$
Q\left(x_{2 j-1}^{(2 n+4)}\right)=g\left(x_{2 j-1}^{(2 n+4)}\right) \quad(j=1,2, \ldots, n+2) .
$$

The following list contains in the left column the lines or groups of consecutive lines which are to be removed from the body of procedure TChex and replaced by the lines from the right column in order to obtain a procedure calculating the coefficients (9), i.e. $a[k]=a_{k}^{(n)}(k=0,1, \ldots, n)$. integer $h, h 2, i 0, i 1, j, k, m, n 1, n 2$; $\quad$ integer $h, h 2, i 0, i 1, j, k, m, n 1, n 11, n 2$;

$$
\begin{array}{ll}
n:=n 2:=h 2:=2 ; & n:=0 ; \\
x:=p ; & n 1:=1 ; \\
a 2:=f ; & \\
a 1:=f ; & \\
x:=q ; & \\
a 0:=f ; & a[0]:=f ; \\
c:=.5 \times(a 0+a 2) ; & \text { for } n 2:=n 1+n 1 \text { while } \\
a[0]:=c+a 1 ; & e x \wedge n 2 \leqslant m \text { do } \\
a[1]:=.5 \times(a 0-a 2) ; & \\
a[2]:=.5 \times(c-a 1) ; & \\
\text { for } n 2:=n 2+n 2 \text { while } & \\
e x \wedge n 2 \leqslant m \text { do } & n 11:=h 2:=n 1+1 ; \\
n 1:=h 2+1 ; & \text { for } j:=n 1 \text { step }-1 \text { until } 0 \text { do } \\
\text { for } j:=h 2 \text { step }-1 \text { until } 1 \text { do } & c 2:=\cos (a 0 \times(j+.5)) ; \\
c 2:=\cos (a 0 \times(j-.5)) ; &
\end{array}
$$


$a[h 2+j]:=f$

$a[i 0]:=c \times(a 0-a 1)$;

$k:=h:=.25 \times n 2$;

$h 2:=n 1-3$;

for $j:=1$ step 1 until $h 2$ do

$i 0:=n 2-h$;

for $k:=i 0-h+1$ step 1 until $i 0$ do

for $j:=k-h 2$ step $-h 2$ until $n 1$ do

$a 2:=1 / n 2 ;$

$a[0]:=.5 \times a[0]$;

for $j:=n 1$ step 1 until $n 2$ do

$a[j]:=a 0-a 1$

$a[0]:=2 \times a[0]$;

$n:=h 2:=n 2$ $a[n 1+j]:=f$

$a[i 0]:=(a 0-a 1) / c$;

$k:=h:=.5 \times n 11$;

for $j:=1$ step 1 until $n$ do

$i 0:=n 1+h 2-1$;

for $k:=n 1+h$ step 1 until $i 0$ do

for $j:=k+h 2$ step $h 2$ until $n 2$ do

$a 2:=.5 / n 11$;

$a[n 1]:=a[n 1] \times(a 2+a 2) ;$

for $j:=n 11$ step 1 until $n 2$ do

$a[j]:=a 1-a 0$

$n 1:=n 2+1$

$n:=n 2$

4. Certification. For the function $f(x)=\log x$ and the interval $\langle p, q\rangle=\left\langle\frac{1}{2}, \frac{3}{2}\right\rangle$ the results given in Table 1 were obtained on the ODRA 1204 computer (accuracy: $2^{-37}$ ). To test the influence of rounding errors, the maximum deviations $\Delta_{n}$ of the approximate coefficients (1) from the appropriate accurate Chebyshev coefficients have been also calculated (Table 2).

TABLE 1

\begin{tabular}{r|r|r|r}
\hline$k$ & \multicolumn{1}{|c|}{$a_{k}^{(2)}$} & \multicolumn{1}{c}{$a_{k}^{(4)}$} & \multicolumn{1}{c}{$a_{k}^{(8)}$} \\
\hline 0 & -.1438410362 & -.1386862144 & -.1386729286 \\
1 & .5493061443 & .5359283009 & .5358983852 \\
2 & -.0719205181 & -.0719205181 & -.0717967711 \\
3 & & .0133778435 & .0128252633 \\
4 & & -.0025774109 & -.0025774109 \\
5 & & & .0005525802 \\
6 & & & -.0001237470 \\
7 & & & .0000299156 \\
8 & & & -.0000066429
\end{tabular}

TABLE 2

\begin{tabular}{r|c}
\hline$n$ & $\Delta_{n}$ \\
\hline 2 & .0134077595 \\
4 & .0005525858 \\
8 & .0000015822 \\
16 & .0000000000 \\
32 & .0000000000 \\
64 & .0000000004 \\
128 & .0000000004 \\
256 & .0000000044 \\
512 & .0000000177 \\
1024 & .0000000926
\end{tabular}

If the actual parameters of procedure TChex are not involved, the calculation time is nearly proportional to $v\left(\log _{2} v+1\right)$, where $v$ is the final value of parameter $n$. 


\section{References}

[1] C. W. Clenshaw, Ohebyshev series for mathematical functions, London 1962.

[2] В. И. Крылов, Приблиюсенное вычисление интегралов, Москва 1959.

[3] Tables of Chebyshev polynomials, Washington 1952.

DEPT. OF NUMERICAL METHODS

MATHEMATICAL INSTITUTE

UNIVERSITY OF WROCEAW

Received on 10. 10. 1970

Z. CYLKOWSKI (Wroolaw

ALGORYTM 13

PRZYBLIŹONE ROZWIJANIE FUNKCJI W SZEREg CZEBYSZE WA

STRESZCZENIE

Procedura TChex dla danej liczby naturalnej $N \geqslant 2$ oblicza przybliżone wartości (1) współczynników rozwinięcia funkcji $f(x)$ w przedziạle $\langle p, q\rangle$ w szereg względem wielomianów Czebyszewa I rodzaju, tj. takie liczby (1), że dla $x \in\langle p, q\rangle$ zachodzi równość (2). Równóść (2) jest dokładna, jeśli $f(x)$ jest wielomianem co najwyżej.n-tego stopnia.

Dane:

$p, q-$ końce przedziału $\langle p, q\rangle$,

$f$ - wyrażenie arytmetyczne o wartości $f(x)$, zależne od parametru $x$,

$n$ - liczba naturalna $N$.

Wyniki:

$n$ - górna granica sumowania w (2), liczba postaci $2^{l}(l$ - liczba naturalna), $a[0: n]$ - tablica przybliżonych wartosci (1) $\left(a[k]=a_{k}^{(n)}\right.$ dla $\left.k=0,1, \ldots, n\right)$. Uwaga: W czasie obliczeń zmienna $n$ przyjmuje kolejno wartości $2,4,8, \ldots$ i dla każdej z nich znajduje się współczynniki (1). Końcowa wartość tej zmiennej jest zawsze nie większa od $N$, ale może zależeć od wartości parametru ex.

Inne parametry:

$x$ - zmienna występująca w czwartym parametrze aktualnym,

ex - wyrażenie boolowskie, które może zależeć od aktualnych wartości $n$ i $a[0: n]$.

Jeśli przy podwojeniu wartości $n$ zachodzi $e x=$ true, to procedura oblicza wspólczynniki (1) dla nowego $n$, a jeśli $e x=$ false, to obliczenia kończy się.

Uwagi:

10 Współczynniki $a_{k}^{(n / 2)}\left(k=0,1, \ldots, \frac{1}{2} n\right)$ można odtworzyó ż wzoru (3). 
$2^{\circ}$ Współczynniki (1) o dolnych wskaźnikach tylko parzystych albo tylko nieparzystych można otrzymać, uwzględniając zależnośc, że jeśli $w$ przedziale $\langle-1,1\rangle$ zachodzi wzór (4), to w przedziale $\langle 0,1\rangle$ zachodzą wzory (5).

Użyta $w$ procedurze TChex metoda jest opisana $w$ \$ 2. Metode tę $i$ treść procedury można zmodyfikować (\$ 3) tak, żeby otrzymać współczynniki (9) rozwinięcia funkcji $f(x)$ w szereg względem wielomianów Czebyszewa II rodzaju. Obliczenia kontrolne (\$ 4) wykonano na maszynie cyfrowej ODRA 1204, przy czym czas obliczeń był proporcjonalny do wielkości $v\left(\log _{2} v+1\right)$, gdzie $v$ jest końcową wartością parametru $n$. 